\title{
Mortalidade e o Tempo de Internação em uma Unidade de Terapia Intensiva Cirúrgica *
}

\section{Mortality and Length of Stay in a Surgical Intensive Care Unit}

Fernando José Abelha ${ }^{1}$; Maria Ana Castro ${ }^{1}$; Nuno Miguel Landeiro ${ }^{1}$; Aida Maria Neves ${ }^{1}$; Cristina Costa Santos ${ }^{2}$

\begin{abstract}
RESUMO
Abelha FJ, Castro MA, Landeiro NM, Neves AM, Santos CC Mortalidade e o Tempo de Internação em uma Unidade de Terapia Intensiva Cirúrgica
\end{abstract}

JUSTIFICATIVA E OBJETIVOS: Em cuidados intensivos os resultados podem ser relacionados aos índices de mortalidade ou morbidade. Quando avaliada de forma isolada, a mortalidade é uma medida insuficiente do resultado na Unidade de Terapia Intensiva (UTI); o tempo de internação pode ser uma medida indireta do resultado relacionado com a morbidade. O objetivo do presente estudo foi avaliar a incidência e os fatores preditivos para mortalidade e tempo de internação dos pacientes admitidos numa UTI cirúrgica.

MÉTODO: Participaram deste estudo prospectivo, realizado, entre abril e julho de 2004, todos os 185 pacientes submetidos a procedimentos programados ou de emergência, admitidos numa UTI cirúrgica. Foram registrados os seguintes parâmetros: idade, sexo, altura e peso, temperatura central estado físico segundo a ASA, tipo de intervenção cirúrgica, porte cirúrgico, técnica anestésica, quantidade e qualidade de fluídos administrados durante a anestesia, monitorização da temperatura ou de técnica de aquecimento corporal peri-operatório, duração da anestesia, tempo de permanência na UTI e no hospital e escore SAPS II.

RESULTADOS: O tempo médio de internação na UTI foi de 4,09 $\pm 10,23$ dias. Fatores de risco significativos para permanências mais prolongadas na UTI foram o valor do escore SAPS II, estado físico ASA, quantidade administrada, durante a intervenção cirúrgica, de colóides, unidades de plasma fresco e unidades de concentrados de hemáceas. Quatorze pacientes $(7,60 \%)$ morreram durante a internação na UTI e 29 (15,70\%) morreram durante a internação hospitalar. Fatores de risco independentes de mortalidade com diferença estatística significativa foram intervenções cirúrgicas de emergência, de grande porte, escores altos SAPS II, permanência prolongada na UTI e no hospital. Fatores protetores com diferença estatística significativa para risco de

\footnotetext{
${ }^{*}$ Recebido do (Received from) Departamento de Anestesiologia e Unidade de Terapia Intensiva da Universidade do Porto, Portugal

1. Consultant in Anesthesiology, Surgical Intensive Care Unit, Department of Anesthesiology and Intensive Care

2. Biostatistics and Medical Informatics Department, Faculty of Medicine at the University of Porto
}

Apresentado (Submitted) em 11 de março de 2005

Aceito (Accepted) para publicação em 21 de outubro de 2005

Endereço para correspondência (Correspondence to)

Dr. Fernando José Abelha

Hospital de São João

Alameda Professor Hernâni Monteiro

4100319 Porto

Portugal

E-mail: abelha@mail.telepac.pt morte hospitalar foram baixo peso corporal e baixo índice de massa corporal (IMC).

CONCLUSÕES: As internações prolongadas em UTI são mais freqüentes nos pacientes mais graves à admissão e estão associadas às maiores mortalidades hospitalares. A mortalidade hospitalar é também mais freqüente em pacientes submetidos a intervenções cirúrgicas de emergência ou de grande porte.

Unitermos: COMPLICAÇÕES: morbidade, mortalidade, pós-operatória; PÓS-OPERATÓRIO: cirurgia de emergência, cirurgia de grande porte; TERAPIA INTENSIVA: mortalidade, tempo de internação

\section{SUMMARY}

Abelha FJ, Castro MA, Landeiro NM, Neves AM, Santos CC Mortality and Length of Stay in a Surgical Intensive Care Unit

BACKGROUND AND OBJECTIVES: Outcome in intensive care can be categorized as mortality related or morbidity related. Mortality is an insufficient measure of ICU outcome when measured alone and length of stay may be seen as an indirect measure of morbidity related outcome. The aim of the present study was to estimate the incidence and predictive factors for intrahospitalar outcome measured by mortality and LOS in patients admitted to a surgical ICU.

METHODS: In this prospective study all 185 patients, who underwent scheduled or emergency surgery admitted to a surgical ICU in a large tertiary university medical center performed during April and July 2004, were eligible to the study. The following variables were recorded: age, sex, body weight and height, core temperature (TC), ASA physical status, emergency or scheduled surgery, magnitude of surgical procedure, anesthesia technique, amount of fluids during anesthesia, use of temperature monitoring and warming techniques, duration of the anesthesia, length of stay in ICU and in the hospital and SAPS II score.

RESULTS: The mean length of stay in the ICU was $4.09 \pm 10.23$ days. Significant risk factors for staying longer in ICU were SAPS II, ASA physical status, amount of colloids, fresh frozen plasma units and packed erythrocytes units used during surgery. Fourteen (7.60\%) patients died in ICU and 29 (15.70\%) died during their hospitalization. Statistically significant independent risk factors for mortality were emergency surgery, major surgery, high SAPS II scores, longer stay in ICU and in the hospital. Statistically significant protective factors against the probability of dying in the hospital were low body weight and low BMI.

CONCLUSIONS: In conclusion, prolonged ICU stay is more frequent in more severely ill patients at admission and it is associated with higher hospital mortality. Hospital mortality is also more frequent in patients submitted to emergent and major surgery.

Key Words: COMPLICATIONS: morbidity, mortality, postoperative; INTENSIVE CARE: mortality, stay length; POSTOPERATIVE PERIOD: emergency surgery, major surgery. 


\section{INTRODUÇÃO}

$\mathrm{O}$ resultados das internações em unidades de terapia intensiva foram inicialmente analisados considerando-se a sobrevida hospitalar e a utilização de recursos, de acordo com a gravidade da doença. Existem vários sistemas de previsão de resultados ${ }^{1-3}$ para pacientes de UTI que são usados rotineiramente em várias UTI de todo o mundo e usam a gravidade das doenças como parâmetro de previsão de mortalidade. Eles têm sido muito usados e seu desempenho já foi bastante estudado em grandes estudos internacionais ${ }^{4}$. Os resultados previstos podem ser usados para tomada de decisão clínica em pacientes individuais e para avaliar a qualidade do atendimento.

Estudos de análise de custos revelaram que o custo diário da UTI por paciente é muito consistente para a maioria dos diagnósticos ${ }^{5}$ e, portanto, o tempo de permanência na UTI (TP) também tem sido usado como medida da utilização de recursos na UTI ${ }^{6,7}$. Apesar dos progressos dos cuidados peri-operatórios, a permanência prolongada na unidade de terapia intensiva ainda é associada a mau prognóstico e a custos mais altos $^{8-10}$

Os fatores de risco, que predispõem à permanência prolongada na UTI após procedimentos cirúrgicos foram identificados e amplamente estudados e são associados com má evolução do paciente e custos elevados ${ }^{11-13}$.

Embora o tempo de permanência na UTI possa ser afetado por políticas de alta hospitalar, por diferentes tipos de práticas e administração dos leitos ${ }^{14}$, a permanência prolongada na UTI pode afetar negativamente o estado de saúde aumentando o risco de infecções, complicações e, possivelmente, a mortalidade ${ }^{15}$. Também afeta a disponibilidade de leitos e poderá resultar em cancelamento de procedimentos cirúrgicos eletivos, levando a longos períodos de espera e tempo perdido na enfermaria antes da admissão à UTI.

O tempo provável de permanência na UTI também pode influenciar as decisões terapêuticas. Estudos recentes indicaram que algumas estratégias terapêuticas com impacto nos resultados podem ter melhor efeito em pacientes com permanência mais longa na UTI ${ }^{16,17}$.

O objetivo deste estudo foi estimar a incidência e os fatores preditivos de mortalidade e tempo de permanência na UTI como medida de resultados intra-hospitalares em pacientes admitidos em UTI cirúrgica.

\section{MÉTODO}

O protocolo foi aprovado pelo Comitê de Ética da instituição. Este estudo prospectivo foi realizado num período de três meses, entre abril e julho de 2004. Todos os pacientes adultos (> 18 anos), submetidos a intervenções cirúrgicas não-cardíacas eletivas ou de emergência e admitidos em UTI cirúrgica de nove leitos de um hospital terciário, participaram do estudo.

As seguintes variáveis clínicas foram registradas na chegada à UTI: idade, sexo, peso corporal e altura, temperatura pré-operatória, estado físico ASA, intervenção cirúrgica ele-

Revista Brasileira de Anestesiologia

Vol. 56, No 1, Janeiro - Fevereiro, 2006 tiva ou de emergência, porte do procedimento cirúrgico, conforme descrição de Kongsayreepong, classificado como grande (cirurgia em que as cavidades corporais ou grandes vasos expostos à temperatura ambiente, tais como grandes procedimentos abdominais, torácicos, vasculares, coluna vertebral torácica com instrumentação ou artroplastia de quadril), médio (cavidades abdominais expostas em menor intensidade como em apendicectomia) e pequeno (superficiais), técnica anestésica, unidades de cristalóides, colóides, concentrado de hemáceas e plasma fresco durante a anestesia, uso de monitor de temperatura e técnicas de aquecimento, e duração da anestesia. A temperatura central (Tc) medida por um termômetro infravermelho de membrana timpânica, foi registrada antes da intervenção cirúrgica na enfermaria, na chegada à UTI e a cada duas horas durante seis horas.

Foram também registrados o TP e a mortalidade na UTI e no hospital, e o Simplified Acute Physiology Score II (SAPS II) foi calculado.

O tempo prolongado de permanência na UTI foi definido como internação por três dias ou mais. Os pacientes foram divididos em dois grupos: pacientes com tempo prolongado de permanência na UTI e pacientes com tempo curto de permanência na UTI. Para a análise da mortalidade, os pacientes também foram divididos em dois grupos: pacientes que morreram durante a permanência no hospital e pacientes que sobreviveram. Os grupos foram comparados para avaliar o relacionamento entre cada variável clínica e o tempo prolongado de permanência na UTI ou a mortalidade, usando análise univariável por regressão logística binária simples com odds ratio (OR) e $95 \%$ IC e teste $t$ para amostras independentes, testes de Qui-quadrado ou Exato de Fisher. Os previsores multivariáveis foram determinados por maior tempo de permanência na UTI e a mortalidade foi determinada por regressão logística binária múltipla com eliminação condicional posterior para examinar os efeitos covariáveis e calcular OR e 95\% IC. O nível significativo de 0,05 foi usado para todas as análises.

As variáveis quantitativas são apresentadas em média \pm DP. Todas as análises foram realizadas com SPSS for Windows (versão 12,0, Chicago, IL).

\section{RESULTADOS}

Cento e oitenta e cinco pacientes participaram do estudo. $O$ TP na UTI variou de 1 a 82 dias, com média \pm DP de 4,09 \pm 10,23 dias. A porcentagem de pacientes que permaneceram na UTI por mais de três dias foi $20 \%(n=37)$ (Tabela I). Os resultados da análise multivariável mostraram que os fatores de risco mais importantes para permanência prolongada na UTI foram SAPS II (OR 1,126, 95\% CI 1,083-1,171, p < 0,001), estado físico ASA(OR 4,764, 95\% CI 1,876-12,096, p $<0,001$ para pacientes estado físico ASAIII/IV), procedimentos de emergência (OR 6,526, 95\% Cl 2,771-15,370, p< $0,001)$, quantidade de colóides, plasma fresco e concentrado eritrócitário usados durante o procedimento (OR 4,954, $95 \%$ Cl 1,514-16,214, $p=0,008$, OR $1,836,95 \%$ Cl 1,163 - 
$2,899, p=0,009$, OR 1,309 95\% Cl 1,014 - 1,689, $p=0,039$, respectivamente). As temperaturas medidas 2, 4 e 6 horas após a internação foram fatores protetores contra a permanência prolongada na UTI (OR 0,602, 95\% Cl 0,392 - 0,923, $p=0,020$, OR 0,622, 95\% Cl 0,390-0,992, p=0,046 e OR $0,454,95 \% \mathrm{Cl} 0,262-0,885, p=0,005$, respectivamente). Os pacientes que permaneceram por mais tempo tiveram mortalidade mais alta na UTI (OR 79,6, 95\% CI 10,0 - 636,9, $p<0,001$ ) e no hospital (OR 14,6, 95\% Cl 5,9 - 36,2, $p<0,001$ ) (Tabela II).

Regressãologística binária múltipla com eliminação condicional posterior para examinar co-variáveis demonstrou que o

Tabela I - Características dos Pacientes $(\mathrm{n}=185)$

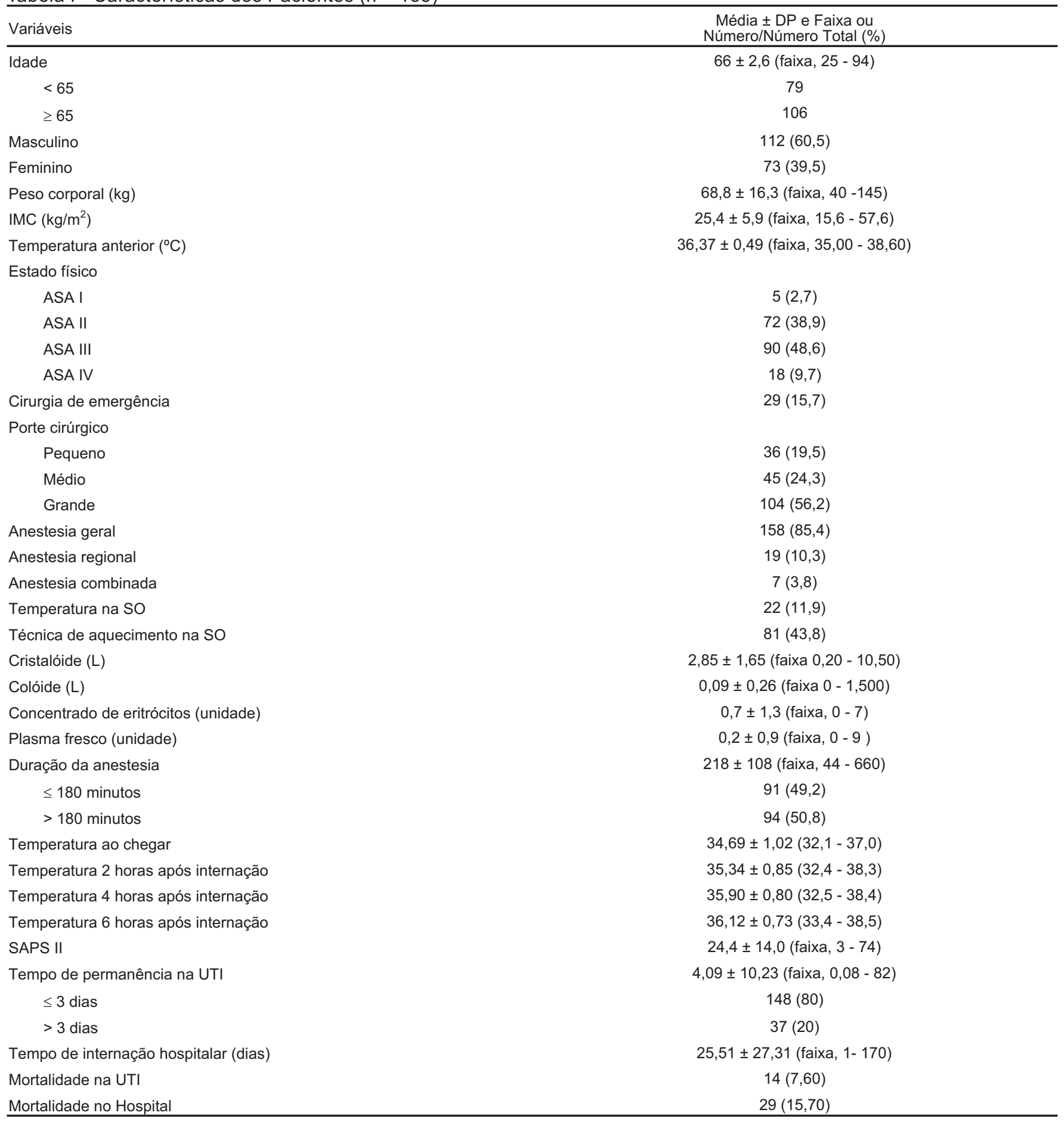


fator mais importante para prever uma permanência prolongada na UTI foram os altos escores SAPS (OR 1,101, 95\% CI $1,053-1,151, p<0,001)$. Esta análise demonstrou que o escore SAPS foi o fator que melhor determinou o TP na UTI (Tabela III).

Quatorze pacientes (7,60\%) morreram na UTI e 29 (15,70\%) durante a internação hospitalar.
De acordo com a análise univariável (Tabela III), idade, sexo, técnica anestésica, uso de técnica de aquecimento peri-operatório, monitoramento da temperatura, duração da anestesia ou do procedimento não foram fatores de risco independentes para mortalidade no hospital. De acordo com a mesma análise, a temperatura não foi um fator de risco na admissão, nem duas, quatro e seis horas após internação na UTI.

Tabela II - Análise Univariável de Previsores Categóricos e Contínuos da Duração da Internação

\begin{tabular}{|c|c|c|c|}
\hline Variáveis & $\begin{array}{c}\text { Permanência UTI } \geq 3 \text { Dias/ } \\
\text { Permanência UTI }<3 \text { Dias } \\
\text { (Número e Média } \pm \text { DP) }\end{array}$ & $\begin{array}{l}\text { Odds Ratio } \\
(95 \% \mathrm{Cl})\end{array}$ & $\mathrm{p}$ \\
\hline \multicolumn{4}{|l|}{ Idade (anos) } \\
\hline$<65$ & $14 / 65$ & 1 & \\
\hline$\geq 65$ & $23 / 83$ & $1,287(0,614-2,695)$ & 0,504 \\
\hline \multicolumn{4}{|l|}{ Sexo } \\
\hline Masculino & $22 / 90$ & $0,945(0,453-1,970)$ & 0,880 \\
\hline Feminino & $15 / 58$ & & \\
\hline Peso corporal (kg) & $69,6 \pm 17,0 / 65,5 \pm 12,8$ & $0,983(0,958-1,007)$ & 0,166 \\
\hline IMC $\left(\mathrm{kg} / \mathrm{m}^{2}\right)$ & $24,1 \pm 4,19 / 25,8 \pm 6,2$ & $0,942(0,875-1,015)$ & 0,116 \\
\hline \multicolumn{4}{|l|}{ Estado físico } \\
\hline ASA I/II & $6 / 71$ & 1 & \\
\hline ASA III/IV & $31 / 77$ & $4,764(1,876-12,096)$ & 0,001 \\
\hline \multicolumn{4}{|l|}{ Cirurgia de emergência/ eletiva } \\
\hline Cirurgia de emergência & $15 / 14$ & $6,526(2,771-15,370)$ & $<0,001$ \\
\hline Cirurgia eletiva & $22 / 134$ & 1 & \\
\hline \multicolumn{4}{|l|}{ Porte cirúrgico } \\
\hline Pequeno & $5 / 31$ & 1 & \\
\hline Médio & $10 / 35$ & $1,663(0,579-4,779)$ & 0,345 \\
\hline Grande & $22 / 82$ & $1,771(0,546-5,750)$ & 0,341 \\
\hline Temperatura na SO & $5 / 17$ & $1,204(0,413-3,508)$ & 0,734 \\
\hline Técnica de aquecimento na SO & $17 / 64$ & $0,896(0,435-1,848)$ & 0,767 \\
\hline Cristalóide (L) & $2,97 \pm 1,77 / 2,82 \pm 1,62$ & $1,055(0,852-1,308)$ & 0,622 \\
\hline Colóide (L) & $0,20 \pm 0,36 / 0,07 \pm 0,22$ & $4,954(1,514-16,214)$ & 0,008 \\
\hline Concentrado de eritrócitos (unidade) & $1,08 \pm 1,61 / 0,59 \pm 1,12$ & $1,309(1,014-1,689)$ & 0,039 \\
\hline Plasma fresco (unidade) & $0,70 \pm 1,80 / 0,12 \pm 0,48$ & $1,836(1,163-2,899)$ & 0,009 \\
\hline Duração da anestesia & $247 \pm 150 / 211 \pm 94$ & & \\
\hline$\leq 180$ minutos & $17 / 74$ & 1 & \\
\hline$>180$ minutos & $20 / 74$ & $1,176(0,571-2,423)$ & 0,659 \\
\hline Temperatura anterior $\left({ }^{\circ} \mathrm{C}\right)$ & $36,46 \pm 0,65 / 36,35 \pm 0,44$ & & \\
\hline Temperatura ao chegar $\left({ }^{\circ} \mathrm{C}\right)$ & $34,44 \pm 1,10 / 34,75 \pm 0,99$ & $0,743(0,520-1,061)$ & 0,102 \\
\hline Temperatura 2 horas após a internação $\left({ }^{\circ} \mathrm{C}\right)$ & $35,04 \pm 1,15 / 35,41 \pm 0,75$ & $0,602(0,392-0,923)$ & 0,020 \\
\hline Temperatura 4 horas após a internação $\left({ }^{\circ} \mathrm{C}\right)$ & $35,66 \pm 1,05 / 35,96 \pm 0,71$ & $0,622(0,390-0,992)$ & 0,046 \\
\hline Temperatura 6 horas após a internação $\left({ }^{\circ} \mathrm{C}\right)$ & $35,80 \pm 1,07 / 36,20 \pm 0,60$ & $0,454(0,262-0,785)$ & 0,005 \\
\hline SAPS II & $40,73 \pm 14,39 / 20,28 \pm 10,54$ & $1,126(1,083-1,171)$ & $<0,001$ \\
\hline Mortalidade na UTI & $13 / 1$ & $79,6(10,0-636,9)$ & $<0,001$ \\
\hline Mortalidade no hospital & $19 / 10$ & $14,6(5,9-36,2)$ & $<0,001$ \\
\hline
\end{tabular}


Tabela III - Análise Univariável de Previsores Categóricos e Contínuos de Mortalidade Hospitalar

\begin{tabular}{|c|c|c|c|}
\hline Variáveis & $\begin{array}{l}\text { Não Sobreviventes/ } \\
\text { Sobreviventes } n^{\circ} \text { ou Média } \pm \text { DP }\end{array}$ & $\begin{array}{l}\text { Odds Ratio } \\
(95 \% \mathrm{Cl})\end{array}$ & $\mathrm{p}$ \\
\hline \multicolumn{4}{|l|}{ Idade (anos) } \\
\hline$<65$ & $14 / 65$ & 1 & \\
\hline$\geq 65$ & $15 / 91$ & $1,307(0,590-2,893)$ & 0,510 \\
\hline \multicolumn{4}{|l|}{ Sexo } \\
\hline Masculino & $19 / 93$ & $0,777(0,339-1,781)$ & 0,551 \\
\hline Feminino & $10 / 63$ & & \\
\hline Peso corporal (kg) & $63,2 \pm 11,8 / 69,8 \pm 16,9$ & $0,970(0,941-0,999)$ & 0,044 \\
\hline $\mathrm{IMC}\left(\mathrm{kg} / \mathrm{m}^{2}\right)$ & $23,1 \pm 3,83 / 25,8 \pm 6,1$ & $0,895(8,15-0,982)$ & 0,019 \\
\hline Estado físico & & & 0,003 \\
\hline ASA I/II & $8 / 69$ & 1 & \\
\hline ASA III/IV & $21 / 87$ & $2,082(0,869-4,987)$ & 0,10 \\
\hline Cirurgia de emergência & $13 / 16$ & $7,109(2,902-17,419)$ & $<0,001$ \\
\hline \multicolumn{4}{|l|}{ Porte cirúrgico } \\
\hline Pequeno & $2 / 34$ & 1 & \\
\hline Médio & $11 / 34$ & $3,091(0,674-14,167)$ & 0,146 \\
\hline Grande & $16 / 88$ & $5,500(1,133-26,69)$ & 0,034 \\
\hline Temperatura na SO & $5 / 17$ & $1,703(0,574-5,052)$ & 0,337 \\
\hline Técnica de aquecimento na SO & $10 / 71$ & $1,587(0,693-3,632)$ & 0,274 \\
\hline Cristalóide (L) & $2,88 \pm 1,59 / 2,85 \pm 1,66$ & $1,015(0,799-1,290)$ & 0,904 \\
\hline Colóide (L) & $0,16 \pm 0,33 / 0,08 \pm 0,24$ & $2,424(0,677-8,683)$ & 0,174 \\
\hline Concentrado eritrocitário (unidade) & $0,83 \pm 1,14 / 0,66 \pm 1,27$ & $1,104(0,824-1,477)$ & 0,508 \\
\hline Plasma fresco (unidade) & $0,17 \pm 0,54 / 0,25 \pm 0,99$ & $0,894(0,524-1,527)$ & 0,683 \\
\hline Duração da anestesia & $204 \pm 112 / 221 \pm 107$ & & \\
\hline$\leq 180$ minutos & $16 / 75$ & 1 & \\
\hline$>180$ minutos & $13 / 81$ & $1,329(0,599-2,948)$ & 0,484 \\
\hline \multicolumn{4}{|l|}{ Temperatura ao chegar } \\
\hline$\leq 35$ & $20 / 87$ & 1 & \\
\hline$>35$ & $9 / 69$ & $0,567(0,243-1,325)$ & 0,190 \\
\hline SAPS II & $41,41 \pm 17,86 / 21,20 \pm 10,54$ & $1,105(1,067-1,144)$ & $<0,001$ \\
\hline \multicolumn{4}{|l|}{ Tempo de permanência na UTI } \\
\hline$<3$ dias & $10 / 138$ & 1 & \\
\hline$\geq 3$ dias & $19 / 18$ & $14,57(5,87-37,18)$ & $<0,001$ \\
\hline Tempo de internação hospitalar (dias) & $45,7 \pm 41,8 / 21,8 \pm 21,9$ & $1,02(1,01-1,04)$ & $<0,001$ \\
\hline
\end{tabular}

Fatores protetores independentes e com diferença estatística significativa para mortalidade foram baixo peso corporal (OR 0,970, 95\% Cl 0,941-0,999, p = 0,044) e baixo índice de massa corpórea (IMC) (OR 0,895, 95\% CI 0,815-0,982, p = 0,019 ), e fatores de risco independentes importantes foram procedimentos de emergência (OR $7,109,95 \% \mathrm{Cl}$ $2,902-17,419, p<0,001)$, de grande porte (OR 5,500,95\% Cl $1,133-26,690, p=0,034)$, escores SAPS II altos (OR 1,105, $95 \% \mathrm{Cl} 1,067-1,144, \mathrm{p}<0,001)$, permanência prolongada na
UTI (OR 14,47, 95\% CI5,87-36,18, p<0,001 para permanência acima de 3 dias) e no hospital (OR 1,024, 95\% Cl 1,011-1,038, $p<0,001$ ).

Aanálise de regressão logística múltipla (Tabela IV) demonstrou que os previsores mais importantes de morte hospitalar foram escores SAPS altos (OR 1,101, 95\% CI 1,053-1,151, p $<0,001$ ), idade (OR 6,541, 95\% Cl 1,685-25,388, $p=0,007$ para pacientes acima de 65 anos) e TP na UTI (OR 3,56, 95\% $\mathrm{Cl} 1,13-11,27 p<0,001)$. A análise demonstrou que esses fo-

Revista Brasileira de Anestesiologia Vol. 56, N 1, Janeiro - Fevereiro, 2006 
ram os fatores que previram de forma significativa a morte intra-hospitalar.

Tabela IV - Previsores de Mortalidade por Regressão Logística Múltipla

\begin{tabular}{lcc}
\hline Variáveis & $\begin{array}{c}\text { Odds Ratio } \\
(95 \% \text { IC })\end{array}$ & $\mathrm{p}$ \\
\hline SAPS II & $1,101(1,053-1,151)$ & $<0,001$ \\
Idade > 65 anos & $6,541(1,685-25,388)$ & 0,007 \\
Tempo de permanência na UTI (dias) & $3,56(1,126-11,265)$ & $<0,001$ \\
\hline
\end{tabular}

\section{DISCUSSÃO}

Nesse estudo, a gravidade dos pacientes, medida pelo estado físico da ASA e pelos escores SAPS II, foi um previsor de permanência prolongada na UTI, o mesmo sendo verdade para pacientes submetidos a procedimentos de emergência e para aqueles que receberam grande quantidade de outros fluidos além dos cristalóides, durante a intervenção cirúrgica. Isso provavelmente reflete o pressuposto de que pacientes mais graves permanecem por mais tempo na UTI. O TP longo na UTI é definido como internação acima de sete dias ${ }^{18,19}$. Devido aos altos custos da UTI e ao fato de que nesse estudo apenas $20 \%$ dos pacientes permaneceram por mais de três dias na UTI, achou-se interessante investigar os previsores clínicos para permanência mais curta na UTI e essa foi a razão de da opção por 3 dias como o ponto de corte para definir permanência prolongada na UTI.

Durante o estudo, os pacientes foram de acordo com os mesmos padrões de tratamento e os protocolos habituais da UTI, incluindo a conduta de tratar a hipotermia de acordo com a evolução do paciente, e isso não influenciou o desfecho nos diferentes grupos.

A hipotermia, à chegada na UTI, não foi um previsor de permanência prolongada, mas a Tc elevada, medida 2, 4 e 6 horas após a internação, foi considerada um fator protetor contra TP longo na UTI. Nesse estudo, os pacientes com permanência prolongada foram quase todos aqueles com complicações pós-operatórias que exigiram tratamento intensivo e aqueles mais gravemente enfermos à admissão.
A mortalidade geral no estudo foi 7,6\% durante permanência na UTI e 15,7\% durante internação hospitalar, Esses valores estão dentro do esperado de acordo com os índices padrão de mortalidade SAPS II.

A instituição sede do estudo possui poucos leitos para terapia semi-intensiva e isso pode explicar o TP mais prolongado na UTI e o fato de que pacientes que permanecem por mais tempo na UTI estão mais propensos ao óbito.

Os previsores clínicos pré-admissão, com diferença estatística significativa para mortalidade foram, principalmente, estado físico ASA, intervenção cirúrgica de emergência e porte cirúrgico. O IMC e peso corporal mais altos foram fatores protetores. Escore SAPS II e TP Iongo na UTI e no hospital foram também fatores independentes de óbito durante a internação.

A classificação ASAé a ferramenta mais utilizada para avaliar o risco dos pacientes durante a anestesia. Embora desenvolvida em 1941 por Saklad ${ }^{20}$, ela continua sendo aceita como padrão para avaliar as condições pré-operatórias.

Vários estudos retrospectivos demonstraram uma correlação entre a classificação ASA e a mortalidade peri-operatória $^{21-24}$ e sugeriram sua utilidade como previsor do desfecho para os pacientes ${ }^{25}$. A importância do tipo de intervenção cirúrgica já foi enfatizada ${ }^{21,24,26,27}$. Intervenções cirúrgicas eletivas ou de curta duração diminuem o risco operatório e existe efeito relativo à desfecho negativo atribuído aos procedimentos de emergência.

Estudos sobre a associação de IMC alto em pacientes internados na UTI e no hospital demonstraram resultados conflitantes. Esse estudo está de acordo com o estudo retrospectivo de Choban ${ }^{28}$, mas não com um estudo recém-publicado de Finkielman e col. ${ }^{29}$ e com outros estudos mais recentes ${ }^{30,31}$ que verificaram o impacto do IMC nos resultados da UTI e demonstraram que IMC alto não estava associado ao alto índice de mortalidade de pacientes no período pós-operatório.

Em conclusão, a permanência prolongada na UTI é mais freqüente em pacientes gravemente enfermos na admissão e está associada a um índice mais alto de mortalidade hospitalar. A mortalidade hospitalar também é freqüente em pacientes submetidos a intervenções cirúrgicas de emergência e de grande porte. 


\section{Mortality and Length of Stay in a Surgical Intensive Care Unit}

Fernando José Abelha, M.D.; Maria Ana Castro, M.D.; Nuno Miguel Landeiro, M.D.; Aida Maria Neves, M.D.; Cristina Costa Santos, M.D.

\section{INTRODUCTION}

Outcome in intensive care have primarily been focused on hospital survival and resource utilization adjusted for severity of illness. Many outcome prediction systems for ICU patients have been developed ${ }^{1-3}$ and are routinely used in many ICU all over the world measuring severity of illness as mortality prediction models. They have been widely used and their performance well studied in large international data set ${ }^{4}$. Predicted outcomes may be used both for clinical decision making in individual patients and for assessing quality of care.

Cost analysis studies have found that the ICU cost per day per patient is remarkably consistent across most diagnose ${ }^{5}$ and therefore, ICU length of stay (LOS) has been also used as a measure of resource utilization in the $\mathrm{ICU}^{6,7}$. Despite refinements in perioperative management, prolonged intensive care unit stay is still associated with poor patient outcome and increased costs ${ }^{8-10}$. Risk factors, which predispose toward prolonged stay in ICU after surgery have been found and widely studied and are associated with poor patient outcome and increased costs ${ }^{11-13}$.

Although LOS in ICU may be affected by discharge policies, variable practice patterns and bed management ${ }^{14}$ prolonged ICU stay can adversely affect the health status by increasing the risk of infection, complications, and, possibly, mortality ${ }^{15}$. It have also impact upon bed availability and could result in cancellation of elective surgeries, leading to long waiting times and time spent on the ward before ICU admission. The likely LOS of a patient may also influence therapeutic decisions. Several recent studies have indicated that some therapeutic strategies that impact on patient outcome may only have an effect on patients with longer ICU stays ${ }^{16,17}$. The aim of the present study was to estimate the incidence and predictive factors for intrahospitalar outcome measured by mortality and LOS in patients admitted to a surgical ICU.

\section{METHODS}

The protocol was approved by our institutional review board. This prospective study was performed during a three-month period between April and July 2004. All postoperative adult patients ( $>18$ years old) who underwent scheduled or emergency noncardiac surgery admitted to a nine bed surgical ICU of a tertiary cares hospital was eligible for the study.

The following clinical variables were recorded on admission to the ICU: age, sex, body weight and height, preoperative body temperature, ASA physical status, emergency or scheduled surgery, magnitude of surgical procedure as de- scribed by Kongsayreepong ${ }^{24}$ and classified in major (surgery in which body cavities or major vessels are exposed to ambient temperature such as major abdominal, thoracic, major vascular, thoracic spine surgery with instrumentation, or hip arthroplasty), medium (surgery in which body cavities are exposed to a lesser degree such as appendectomy), and minor surgery (superficial surgery), anesthesia technique, amount of crystalloids, colloids, packed erythrocytes and fresh frozen plasma during anesthesia, use of temperature monitoring and warming techniques, and duration of the anesthesia. Core temperature (Tc) measured by an infrared tympanic membrane thermometer was evaluated before surgery in the ward, on arrival at the ICU and every two hours until 6 hours after.

For all patients we also recorded the LOS and the mortality in the ICU and in the hospital and the Simplified Acute Physiology Score II (SAPS II) was calculated ${ }^{18}$.

Postoperative prolonged ICU stay was defined as intensive care lasting for three days and longer. Two groups of patients were created: patients with prolonged ICU stay and patients with no prolonged ICU stay. For mortality analysis we also created two groups of patients: patients who died during their stay in the hospital and patients who survived. Groups were compared to assess the relationship between each clinical variable and long ICU stay or mortality using univariate analysis performed by simple binary logistic regression with an odds ratio (OR) and its $95 \% \mathrm{Cl}$ and independent sample test, Chi-square or Fisher's Exact tests. Multivariate predictors were determined for staying longer in ICU and mortality by multiple regression binary logistic with forward conditional elimination to examine covariate effects of each and calculate OR and their $95 \% \mathrm{Cl}$. A two-sided significance level of 0.05 was used for all analysis.

Quantitative variables are presented as mean \pm SD. All analyses were performed using SPSS for Windows (version 12.0, Chicago, IL).

\section{RESULTS}

All 185 patients were admitted in the study. The LOS in the ICU varied from 1 to 82 days (Table II) with a mean \pm SD of $4.09 \pm 10.23$ days (Table I). The percentage of patients who stayed in ICU longer than 3 days were $20 \%(n=37)($ Table I). According to univariate analysis significant risk factors for staying longer in ICU were SAPS II (OR 1.126, 95\% CI 1.083-1.171, p<0.001), ASA physical status (OR 4.764, 95\% Cl 1.876-12.096, $p<0.001$ for ASA III/IV patients), emergency surgery (OR 6.526, 95\% Cl 2.771-15.370, $p<0.001$ ), amount of colloids, fresh frozen plasma and packed erythrocytes used during surgery (OR 4.954, 95\% Cl $1.514-16.214, p=0.008$, OR $1.836,95 \% \mathrm{Cl} 1.163-2.899, p=$ 0.009 , OR $1.30995 \% \mathrm{Cl} 1.014-1.689, p=0.039$, respectively). Temperatures measured at 2, 4 and 6 hours after admission were protective factors against staying longer in ICU (OR 0.602, 95\% Cl 0.392-0.923, p = 0.020, OR 0.622, 95\% Cl $0.390-0.992, p=0.046$ and OR $0.454,95 \%$ Cl $0.262-0.885, p$ $=0.005$, respectively). Patients who stayed longer in ICU sig- 
Table I - Patient Baseline Characteristics ( $\mathrm{n}=185)$

\begin{tabular}{|c|c|}
\hline Variables & $\begin{array}{l}\text { Mean } \pm \text { SD and Range or } \\
\text { Number/Total Number }(\%)\end{array}$ \\
\hline Age & $66.0 \pm 2.6($ range, $25-94)$ \\
\hline$<65$ & 79 \\
\hline$\geq 65$ & 106 \\
\hline Male & $112(60.5)$ \\
\hline Female & $73(39.5)$ \\
\hline Body weight (kg) & $68.8 \pm 16.3($ range, $40-145)$ \\
\hline BMI $\left(\mathrm{kg} / \mathrm{m}^{2}\right)$ & $25.4 \pm 5.9$ (range, $15.6-57.6)$ \\
\hline Previous temperature $\left({ }^{\circ} \mathrm{C}\right)$ & $36.37 \pm 0.49$ (range, $35.00-38.60)$ \\
\hline \multicolumn{2}{|l|}{ Physical status } \\
\hline ASA I & $5(2.7)$ \\
\hline ASA II & $72(38.9)$ \\
\hline ASA III & $90(48.6)$ \\
\hline ASA IV & $18(9.7)$ \\
\hline Emergency surgery & $29(15.7)$ \\
\hline \multicolumn{2}{|l|}{ Magnitude of surgery } \\
\hline Minor & $36(19.5)$ \\
\hline Medium & $45(24.3)$ \\
\hline Major & $104(56.2)$ \\
\hline General anesthesia & $158(85.4)$ \\
\hline Regional anesthesia & $19(10.3)$ \\
\hline Combined anesthesia & $7(3.8)$ \\
\hline Temperature monitoring in OR & $22(11.9)$ \\
\hline Warming technique in OR & $81(43.8)$ \\
\hline Intravenous crystalloids (L) & $2.85 \pm 1.65$ (range $0.20-10.50$ ) \\
\hline Intravenous colloids (L) & $0.09 \pm 0.26($ range $0-1,500)$ \\
\hline Packed erythrocytes (Units) & $0.7 \pm 1.3($ range, $0-7)$ \\
\hline Fresh frozen plasma (Units) & $0.2 \pm 0.9($ range, $0-9)$ \\
\hline Duration of anesthesia & $218 \pm 108$ (range, $44-660$ ) \\
\hline$\leq 180$ minutes & $91(49.2)$ \\
\hline$>180$ minutes & $94(50.8)$ \\
\hline Temperature on admission & $34,69 \pm 1,02(32,1-37,0)$ \\
\hline Temperature 2 hours after admission & $35,34 \pm 0,85(32,4-38,3)$ \\
\hline Temperature 4 hours after admission & $35,90 \pm 0,80(32,5-38,4)$ \\
\hline Temperature 6 hours after admission & $36,12 \pm 0,73(33,4-38,5)$ \\
\hline SAPS II & $24.4 \pm 14.0($ range $3-74)$ \\
\hline Length of ICU stay & $4.09 \pm 10.23$ (range, $0.08-82$ ) \\
\hline$\leq 3$ days & $148(80)$ \\
\hline$>3$ days & $37(20)$ \\
\hline Length of hospital stay (days) & $25.51 \pm 27.31$ (range $1-170$ ) \\
\hline Mortality in the ICU & $14(7.60)$ \\
\hline Mortality in the Hospital & $29(15.70)$ \\
\hline
\end{tabular}


nificantly have a higher mortality in the ICU (OR 79.6, $95 \% \mathrm{CI}$ $10.0-636.9, \mathrm{p}<0.001)$ and in the hospital (OR 14.6, 95\% Cl 5.9 - 36.2, $p<0.001$ ) (Table II).

Multiple regression binary logistic with forward conditional elimination to examine all covariate effects of each factor showed considerably significant factor predicting longer staying in the ICU to be higher SAPS scores (OR 1.101, 95\% $\mathrm{Cl} 1.053-1.151, \mathrm{p}<0.001)$. This analysis showed that SAPS score was the factor that more significantly predicts longer LOS in ICU (Table III).

Table II - Univariate Analysis of Categorical and Continuous Predictors of Length of Stay

\begin{tabular}{|c|c|c|c|}
\hline Variables & $\begin{array}{c}\text { ICU Stay } \geq 3 \text { Days / } \\
\text { ICU Stay }<3 \text { Days } \\
\text { (Number or Mean } \pm \text { SD) }\end{array}$ & $\begin{array}{l}\text { Odds Ratio } \\
(95 \% \mathrm{Cl})\end{array}$ & $\mathrm{p}$ - value \\
\hline \multicolumn{4}{|l|}{ Age (years) } \\
\hline$<65$ & $14 / 65$ & 1 & \\
\hline$\geq 65$ & $23 / 83$ & $1.287(0.614-2.695)$ & 0.504 \\
\hline \multicolumn{4}{|l|}{ Gender } \\
\hline Male & $22 / 90$ & $0.945(0.453-1.970)$ & 0.880 \\
\hline Female & $15 / 58$ & & \\
\hline Body weight $(\mathrm{kg})$ & $69.6 \pm 17.0 / 65.5 \pm 12.8$ & $0.983(0.958-1.007)$ & 0.166 \\
\hline $\operatorname{BMI}\left(\mathrm{kg} / \mathrm{m}^{2}\right)$ & $24.1 \pm 4.19 / 25.8 \pm 6.2$ & $0.942(0.875-1.015)$ & 0.116 \\
\hline \multicolumn{4}{|l|}{ Physical status } \\
\hline ASA I/II & $6 / 71$ & 1 & \\
\hline ASA III/IV & $31 / 77$ & $4.764(1.876-12.096)$ & 0.001 \\
\hline \multicolumn{4}{|l|}{ Emergency /elective surgery } \\
\hline Emergency surgery & $15 / 14$ & $6.526(2.771-15.370)$ & $<0.001$ \\
\hline Elective surgery & $22 / 134$ & 1 & \\
\hline \multicolumn{4}{|l|}{ Magnitude of surgery } \\
\hline Minor & $5 / 31$ & 1 & \\
\hline Medium & $10 / 35$ & $1.663(0.579-4.779)$ & 0.345 \\
\hline Major & $22 / 82$ & $1.771(0.546-5.750)$ & 0.341 \\
\hline Temperature monitoring in $\mathrm{OR}$ & $5 / 17$ & $1.204(0.413-3.508)$ & 0.734 \\
\hline Warming technique in OR & $17 / 64$ & $0.896(0.435-1.848)$ & 0.767 \\
\hline Intravenous crystalloids (L.) & $2.97 \pm 1.77 / 2.82 \pm 1.62$ & $1.055(0,852-1.308)$ & 0.622 \\
\hline Intravenous colloids (L.) & $0.20 \pm 0.36 / 0.07 \pm 0.22$ & $4.954(1.514-16.214)$ & 0.008 \\
\hline Packed erythrocytes (Units) & $1.08 \pm 1.61 / 0.59 \pm 1.12$ & $1.309(1.014-1.689)$ & 0.039 \\
\hline Fresh frozen plasma (Units) & $0.70 \pm 1.80 / 0.12 \pm 0.48$ & $1.836(1.163-2.899)$ & 0.009 \\
\hline Duration of anesthesia & $247 \pm 150 / 211 \pm 94$ & & \\
\hline$\leq 180$ minutes & $17 / 74$ & 1 & \\
\hline$>180$ minutes & $20 / 74$ & $1.176(0.571-2.423)$ & 0.659 \\
\hline Previous temperature $\left({ }^{\circ} \mathrm{C}\right)$ & $36.46 \pm 0.65 / 36.35 \pm 0.44$ & & \\
\hline Temperature at admission $\left({ }^{\circ} \mathrm{C}\right)$ & $34.44 \pm 1.10 / 34.75 \pm 0.99$ & $0.743(0.520-1.061)$ & 0.102 \\
\hline Temperature 2 hours after admission $\left({ }^{\circ} \mathrm{C}\right)$ & $35.04 \pm 1.15 / 35.41 \pm 0.75$ & $0.602(0.392-0.923)$ & 0.020 \\
\hline Temperature 4 hours after admission $\left({ }^{\circ} \mathrm{C}\right)$ & $35.66 \pm 1.05 / 35.96 \pm 0.71$ & $0.622(0.390-0.992)$ & 0.046 \\
\hline Temperature 6 hours after admission $\left({ }^{\circ} \mathrm{C}\right)$ & $35.80 \pm 1.07 / 36.20 \pm 0.60$ & $0.454(0.262-0.785)$ & 0.005 \\
\hline SAPS II & $40.73 \pm 14.39 / 20.28 \pm 10.54$ & $1.126(1.083-1.171)$ & $<0.001$ \\
\hline Mortality in the ICU & $13 / 1$ & $79.6(10.0-636.9)$ & $<0.001$ \\
\hline Mortality in the Hospital & $19 / 10$ & $14.6(5.9-36.2)$ & $<0.001$ \\
\hline
\end{tabular}


Table III - Univariate Analysis of Categorical and Continuous Predictors of Mortality in the Hospital

\begin{tabular}{|c|c|c|c|}
\hline Variables & $\begin{array}{l}\text { Nonsurvivors / } \\
\text { Survivors } \mathrm{n}^{\circ} \text { or Mean } \pm \mathrm{SD}\end{array}$ & $\begin{array}{l}\text { Odds Ratio } \\
(95 \% \mathrm{Cl})\end{array}$ & $\mathrm{p}$ \\
\hline \multicolumn{4}{|l|}{ Age (years) } \\
\hline$<65$ & $14 / 65$ & 1 & \\
\hline$\geq 65$ & $15 / 91$ & $1.307(0.590-2.893)$ & 0.510 \\
\hline \multicolumn{4}{|l|}{ Gender } \\
\hline Male & $19 / 93$ & $0.777(0.339-1.781)$ & 0.551 \\
\hline Female & $10 / 63$ & & \\
\hline Body weight (kg) & $63.2 \pm 11.8 / 69.8 \pm 16.9$ & $0.970(0.941-0.999)$ & 0.044 \\
\hline BMI $\left(\mathrm{kg} / \mathrm{m}^{2}\right)$ & $23.1 \pm 3.83 / 25.8 \pm 6.1$ & $0.895(8.15-0.982)$ & 0.019 \\
\hline Physical status & & & 0.003 \\
\hline ASA I/II & $8 / 69$ & 1 & \\
\hline ASA III/IV & $21 / 87$ & $2.082(0.869-4.987)$ & 0.10 \\
\hline Emergency surgery & $13 / 16$ & $7.109(2.902-17.419)$ & $<0.001$ \\
\hline \multicolumn{4}{|l|}{ Magnitude of surgery } \\
\hline Minor & $2 / 34$ & 1 & \\
\hline Medium & $11 / 34$ & $3.091(0.674-14.167)$ & 0.146 \\
\hline Major & $16 / 88$ & $5.500(1.133-26.69)$ & 0.034 \\
\hline Temperature monitoring in OR & $5 / 17$ & $1.703(0.574-5.052)$ & 0.337 \\
\hline Warming technique in OR & $10 / 71$ & $1.587(0.693-3.632)$ & 0.274 \\
\hline Intravenous crystalloids (L) & $2.88 \pm 1.59 / 2.85 \pm 1.66$ & $1.015(0,799-1.290)$ & 0.904 \\
\hline Intravenous colloids (L) & $0.16 \pm 0.33 / 0.08 \pm 0.24$ & $2.424(0.677-8.683)$ & 0.174 \\
\hline Packed erythrocytes (Units) & $0.83 \pm 1.14 / 0.66 \pm 1.27$ & $1.104(0.824-1.477)$ & 0.508 \\
\hline Fresh frozen plasma (Units) & $0.17 \pm 0.54 / 0.25 \pm 0.99$ & $0.894(0.524-1.527)$ & 0.683 \\
\hline Duration of anesthesia & $204 \pm 112 / 221 \pm 107$ & & \\
\hline$\leq 180$ minutes & $16 / 75$ & 1 & \\
\hline$>180$ minutes & $13 / 81$ & $1.329(0.599-2.948)$ & 0.484 \\
\hline \multicolumn{4}{|l|}{ Temperature at admission } \\
\hline$\leq 35$ & $20 / 87$ & 1 & \\
\hline$>35$ & $9 / 69$ & $0.567(0.243-1.325)$ & 0.190 \\
\hline SAPS II & $41.41 \pm 17.86 / 21.20 \pm 10.54$ & $1.105(1.067-1.144)$ & $<0.001$ \\
\hline \multicolumn{4}{|l|}{ Length of ICU stay } \\
\hline$<3$ days & $10 / 138$ & 1 & \\
\hline$\geq 3$ days & $19 / 18$ & $14.57(5.87-37.18)$ & $<0.001$ \\
\hline Length of hospital stay (days) & $45.7 \pm 41.8 / 21.8 \pm 21.9$ & $1.02(1.01-1.04)$ & $<0.001$ \\
\hline
\end{tabular}

Fourteen (7.60\%) patients died in ICU and 29 (15.70\%) died during their hospitalization.

According to univariate analysis (Table III), age, sex, anesthesia technique, use of a perioperative warming technique, temperature monitoring, duration of anesthesia or surgery were not independent risk factors for mortality in the hospital. In the same analysis, temperature was not a risk factor at admission, neither at two, four and six hours after arrival in ICU.

Statistically significant independent protective factors for mortality were low body weight (OR 0.970, 95\% Cl 0.941-0.999, p=

Revista Brasileira de Anestesiologia

Vol. 56, № 1, Janeiro - Fevereiro, 2006
0.044 ) and low Body Mass Index (BMI) (OR 0.895, 95\% Cl $0.815-0.982, p=0.019)$, and statistically significant independent risk factors were emergency surgery (OR 7.109, 95\% Cl 2.902-17.419, $\mathrm{p}<0.001$ ), major surgery (OR 5.500, 95\% Cl 1.133-26.690, $p=0.034$ ), high SAPS II scores (OR 1.105, 95\% Cl 1.067-1.144, p < 0.001), longer stay in ICU (OR 14.47, 95\% CI 5.87-36.18, $p<0.001$ for LOS longer than 3 days) and in the hospital (OR 1.024, 95\% Cl 1.011-1.038, p < 0.001).

The multiple logistic regression analysis (Table IV) showed that considerably significant factors predicting death in the 
hospital were higher SAPS scores (OR 1.101, 95\% Cl $1.053-1.151, p<0.001$ ), age (OR 6.541, 95\% Cl $1.685-25.388, p=0.007$ for patients older than 65 years) and LOS in the ICU (OR 3.56, 95\% CI 1.13-11.27 p<0.001). The analysis showed that these were the factors that more significantly predicted death in the intrahospitalar setting.

Table IV - Predictors of Mortality by Multiple Logistic Regression

\begin{tabular}{lcc}
\multicolumn{1}{c}{ sion } & $\begin{array}{c}\text { Odds Ratio } \\
(95 \% \mathrm{Cl})\end{array}$ & $\mathrm{p}$ \\
\hline Variables & $1.101(1.053-1.151)$ & $<0.001$ \\
\hline SAPS II & $6.541(1.685-25.388)$ & 0.007 \\
Age $>65$ years & $3.56(1.126-11.265)$ & $<0.001$ \\
\hline
\end{tabular}

\section{DISCUSSION}

In this study, severity of illness of patients as measured by ASA physical status and SAPS II scores were predictors of prolonged stay in ICU and the same was true for emergent surgical patients and those with greater amount of intraoperative intravenous fluid administration other than crystalloids. This probably reflects the assumption that more severely ill patients stay longer in ICU.

Long ICU LOS has been defined as stay of more than 7 days 18,19. As a result of high cost of ICU stay, and because in our study only $20 \%$ of patients stayed longer than 3 days in ICU we thought it was of interest to find clinical predictors for more restricted LOS in ICU and in fact those were the reasons to choose 3 days as the cut-off point to consider long ICU stay. During this study, all patients were managed using the same standard of care and with ICU habitual protocols, including that of hypothermia treatment being applied according to the patient's evolution and so this did not influence outcome in the different groups.

Hypothermia at ICU admission was not predictor of staying longer in the ICU but higher Tc measured at 2, 4 and 6 hours after arrival were found to be protective factors for longer ICU LOS.

In our study patients with prolonged ICU stay included almost all patients that had postoperative complications that lead them to increased requirements for intensive care treatment and those who were more severely ill at admission.

Overall mortality in our study was 7,6\% during ICU stay and $15,7 \%$ during hospital stay. These values are in the range of what was expected by the correspondent standard mortality ratios of SAPS II.

In our institution, there are few beds providing intermediate care and that could explain longer LOS in ICU and the fact that patients who stay longer in ICU are more prone to die.

Statistically significant preadmission clinical predictors of death were ASA physical status; emergency surgery and magnitude of surgery being higher BMI and body weight were protective factors. SAPS II score and LOS in ICU and in the hospital were also statistically independent factors for dying during hospital stay.
The ASA classification has established itself as the most widely used patient risk assessment tool in anesthesia. Although developed in 1941 by Saklad ${ }^{20}$ it remains accepted as a standard for assessing preoperative fitness.

Several retrospective studies have demonstrated a correlation between ASA classification and perioperative mortality $21-24$ and have suggested its usefulness as a predictor of patient outcome ${ }^{25}$.

The importance of the type of surgery has been emphasized previously ${ }^{21,24,26,27}$. Elective surgery and minor surgery reduce operative risk and there is an effect on poor outcome attributable to emergency surgery.

Studies addressing the association of high BMI in patients admitted to the ICU and the hospital have demonstrated conflicting results. Our study agree with Choban retrospective study ${ }^{28}$ but not with a recently published study by Finkielman et al. ${ }^{29}$ and with other recent studies ${ }^{30,31}$ that have investigated the impact of BMI on ICU outcome and have showed that high $\mathrm{BMI}$ was not associated with high mortality in post-operative patients.

In conclusion, prolonged ICU stay is more frequent in more severely ill patients at admission and is associated with higher hospital mortality. Hospital mortality is also more frequent in patients submitted to emergent surgery and major surgery.

\section{REFERENNCIAS - REFERENCES}

01. Le Gall JR, Loirat P, Alperovitch A et al - A simplified acute physiology score for ICU patients. Crit Care Med, 1984;12:975-977.

02. Knaus WA, Draper EA, Wagner DP et al - APACHE II: a severity of disease classification system. Crit Care Med, 1985;13:818-829.

03. Knaus WA, Wagner DP, Draper EA et al - The APACHE III prognostic system. Risk prediction of hospital mortality for critically ill hospitalized adults. Chest, 1991;100:1619-1636.

04. Castella X, Artigas A, Bion J et al - A comparison of severity of illness scoring systems for intensive care unit patients: results of a multicenter, multinational study. Crit Care Med, 1995;23: 1327-1335.

05. Noseworthy TW, Konopad E, Shustack A et al - Cost accounting of adult intensive care: methods and human and capital inputs. Crit Care Med, 1996;24:1168-1172.

06. Wong DT, Gomez M, McGuire GP et al - Utilization of intensive care unit days in a Canadian medical-surgical intensive care unit. Crit Care Med, 1999;27:1319-1324.

07. Knaus WA, Wagner DP, Zimmerman JE et al - Variations in mortality and length of stay in intensive care units. Ann Intern Med, 1993;118:753-761.

08. Ryan TA, Rady MY, Bashour CA et al - Predictors of outcome in cardiac surgical patients with prolonged intensive care stay. Chest, 1997;112:1035-1042.

09. Tuman KJ, McCarthy RJ, March RJ et al - Morbidity and duration of ICU stay after cardiac surgery. A model for preoperative risk assessment. Chest, 1992;102:36-44.

10. Rosenberg AL, Watts C - Patients readmitted to ICUs: a systemic review of risk factors and outcomes. Chest, 2000;118: 492-502.

11. Hammermeister KE - Risk, predicting outcomes, and improving care. Circulation, 1995;91:899-900. 
12. Bucerius J, Gummert JF, Walther T et al - Predictors of prolonged ICU stay after on-pump versus off-pump coronary artery bypass grafting. Intensive Care Med, 2004;30:88-95.

13. Tu JV, Jaglal SB, Naylor CD - Multicenter validation of a risk index for mortality, intensive care unit stay, and overall hospital length of stay after cardiac surgery. Steering Committee of the Provincial Adult Cardiac Care Network of Ontario. Circulation, 1995;91:677-684.

14. Predicting outcome in ICU patients. $2^{\text {nd }}$ European Consensus Conference in Intensive Care Medicine. Intensive Care Med, 1994;20:390-397.

15. Gilio AE, Stape A, Pereira CR et al - Risk factors for nosocomial infections in a critically ill pediatric population: a 25 -month prospective cohort study. Infect Control Hosp Epidemiol, 2000;21:340-342.

16. van den Berghe $G$, Wouters $P$, Weekers $F$ et al - Intensive insulin therapy in the critically ill patients. N Eng J Méd, 2001;345:1359-1367.

17. Corwin HL, Gettinger A, Rodriguez RM et al - Efficacy of recombinant human erythropoietin in the critically ill patient: a randomized, double-blind, placebo-controlled trial. Crit Care Med, 1999;27:2346-2350.

18. Le Gall JR, Lemeshow S, Saulnier F - A new Simplified Acute Physiology Score (SAPSII) based on a European/North American multicenter study. JAMA, 1993;270:2957-2963.

19. Stricker K, Rothen HU, Takala J - Resource use in the ICU: shortvs. long-term patients. Acta Anaesthesiol Scand, 2003;47: 508-515.

20. Saklad M - Grading of patients for surgical procedures. Anesthesiology, 1941;2:281-284.

21. Cook TM, Day CJ - Hospital mortality after urgent and emergency laparotomy in patients aged $65 \mathrm{yr}$ and over. Risk and prediction of risk using multiple logistic regression analysis. $\mathrm{Br} \mathrm{J}$ Anaesth, 1998;80;776-781.

22. Wolters U, Wolf $\mathrm{T}$, Stutzer $\mathrm{H}$ et al - ASA classification and perioperative variables as predictors of postoperative outcome. Br J Anaesth, 1996;77:217-222.

23. Pedersen T, Eliasen $\mathrm{K}$, Ravnborg $\mathrm{M}$ et al - Risk factors, complications and outcome in anaesthesia. A pilot study. Eur $\mathrm{J}$ Anaesthesiol, 1986;3:225-239.

24. Kongsayreepong S, Chaibundit C, Chadpaibool J et al - Predictor of core hypothermia and surgical intensive care unit, Anesth Analg, 2003;96:826-833.

25. Donati A, Ruzzi M, Adrario E et al - A new and feasible model for predicting operative risk. Br J Anaesth, 2004;93:393-399.

26. Arvidsson S, Ouchterlony J, Sjostedt L et al - Predicting postoperative adverse events. Clinical efficiency of four general classification systems. The project perioperative risk Acta Anaesthesiol Scand, 1996;40:783-791.

27. Tiret L, Hatton F, Desmonts JM et al - Prediction of outcome of anaesthesia in patients over 40 years: a multifactorial risk index. Stat Med, 1988;7:947-954.

28. Choban PS, Weireter LJ, Maynes C - Obesity and increased mortality in blunt trauma. J Trauma, 1991;31:1253-1257.

29. Finkielman JD, Gajic O, Afessa B - Underweight is independently associated with mortality in post-operative and non-operative patients admitted to the intensive care unit: a retrospective study. BMC Emerg Med, 2004;4:3.

30. Tremblay A, Bandi $V$ - Impact of body mass index on outcomes following critical care. Chest, 2003;123:1202-1207.

31. Garrouste-Orgeas M, Troche G, Azoulay E et al - Body mass index. An additional prognostic factor in ICU patients. Intensive Care Med, 2004;30:437-443.

\section{RESUMEN}

Abelha FJ, Castro MA, Landeiro NM, Neves AM, Santos CC Mortalidad y Tiempo de Internación en una Unidad de Terapia Intensiva Quirúrgica

JUSTIFICATIVA E OBJETIVOS: En cuidados intensivos los resultados pueden ser relacionados con índices de mortalidad o morbilidad. Cuando se evalúa de forma aislada, la mortalidad es una medida insuficiente de los resultados de una Unidad de Terapia Intensiva (UTI); el tiempo de internación puede ser una medida indirecta de resultados relacionados con la morbilidad. El objetivo del presente estudio fue evaluar la incidencia y los factores predictivos para mortalidad y tiempo de internación de los pacientes admitidos en una UTI quirúrgica.

MÉTODO: Participaron en este estudio prospectivo, realizado entre abril y julio de 2004, todos los 185 pacientes sometidos a procedimientos programados o de emergencia, admitidos en la UTI quirúrgica. Fueron registrados los siguientes parámetros: edad, sexo, altura y peso, temperatura central, estado físico según la ASA, tipo de intervención quirúrgica, porte quirúrgico, técnica anestésica, cantidad y calidad de fluidos administrados durante la anestesia, monitorización de la temperatura o la técnica de calentamiento corporal perioperatorio, duración de la anestesia, tiempo de permanencia en la UTI y en el hospital y escore SAPS II.

RESULTADOS: El tiempo medio de internación en la UTI fue de $4,09 \pm 10,23$ días. Factores de riesgo significativos para permanencia más prolongada en la UTI fueron el valor del escore SAPS II, estado físico ASA, cantidad de coloides administrada durante la intervención quirúrgica, unidades de plasma fresco y unidades de concentrados de hematíes. Catorce pacientes $(7,60 \%)$ murieron durante la internación en la UTI y otros 29 (15,70\%) murieron durante la internación hospitalar. Factores de riesgo independientes de mortalidad con diferencia estadística significativa fueron intervenciones quirúrgicas de emergencia, de gran porte, escores altos SAPS II, permanencia prolongada en la UTI y en el hospital. Factores protectores con diferencia estadística significativa para riesgo de muerte hospitalarfueron bajo peso corporal y bajo índice de masa corporal (IMC).

CONCLUSIONES: Las internaciones prolongadas en UTI son más frecuentes en los pacientes más graves en el momento de la admisión y están asociadas a mayor mortalidad hospitalar. La mortalidad hospitalar es también más frecuente en pacientes sometidos a intervenciones quirúrgicas de emergencia o de porte mayor. 